\title{
Radical Prostatectomy versus External Beam Radiotherapy - Indication and the Way to the Decision: A Cumulative Non- Interventional Unicentric Retrospective Trial of about 20 Years
}

\author{
Wolf-Diether U Boehm ${ }^{1^{*}}$, Rainer Koch ${ }^{2}$, Michael Froehner ${ }^{5}$, Stefanie Wenzel ${ }^{4}$, Carmen Werner ${ }^{3}$, Alex- \\ ander Mehnert ${ }^{3}$ and Manfred P Wirth ${ }^{4}$
}

\author{
${ }^{1}$ Academic Student Training, Private Consultation for Urology, Technical University Dresden, Germany \\ ${ }^{2}$ Institute for Biometry and Medical Statistics, Technical University Dresden, Germany \\ ${ }^{3}$ Clinical Cancer Register of Dresden, Germany \\ ${ }^{4}$ Clinic and Outpatient Department for Urology, Technical University Dresden, Germany \\ ${ }^{5}$ Clinic for Urology, Zeisigwaldclinicum Bethanien Chemnitz, Germany
}

*Corresponding author: Wolf-Diether U Boehm, Academic Student Training, Private Consultation for Urology, Technical University Dresden, Comeniusstr. 50a, 01309 Dresden, Germany, Tel: +49-1629-211-296

\begin{abstract}
Design, setting: From the clinical reality of a private consultation, a non-interventional, parallel, two-armed, non-randomized study was started in close cooperation with the Regional Clinical Cancer Register Dresden for the period 1996-2016, in order to objectively define the allocation algorithms for the primary therapy decision RPE vs. ERBT retrospectively in the curative setting for clinically, localized to locally advanced tumor. Furthermore, the implications of this decision on the result should be reviewed retrospectively in terms of multiple factors. For this purpose, univariate and multivariate models were sought based on clinically relevant predictors (variables), which from a large practice's perspective could have a different prognostic significance for the two alternative therapy options.

A total of 742 patients were included in the study chronologically after biopsy confirmation/staging. At the follow-up, the study participants were only subject to guideline-compliant $[1,2]$, routine-based, diagnostic and course-based individual therapeutic regulations in accordance with GCP. The patients' written consent to participate in the study, data storage and anonymized scientific processing was obtained immediately after the diagnosis before enrollment.
\end{abstract}

Results: Variable for the allocation of therapy alternatives RPE ( $n$ 465) vs. EBRT ( $n$ 234) are age ( $p<0.0001$ ), PSA ( $p$ 0.43390), Gleason score total $(p<0.001)$, D'Amico index $(p<0.001)$, Charlson index $(p<0.001)$, biopsy score $(p$ $0.4612)$ and the volume $(p<0.0001)$ - here in a univariate comparison. The prediction precision at $89.6 \%$ is a good argument against a non-evidence decision.

Overall survival (n 734 Patients, 20 years/all risks): RPE $56.8 \%$ and EBRT $19.2 \%$. Relapses defined as per [2] in PSA/local/systemic situation in the RPE file $25.87 \% / 4.48 \% /$ $0.61 \%$, - and in the EBRT file $18.15 \% / 4.03 \% / 4.03 \%$.

In the competing risk analysis for cancer-specific death (CMR), on the other hand, between the two files (RPE $16.2 \% / E B R T 20.5 \%$ ) post 20 years, there was no significant difference ( $p=0.2122)$ - in contrast to the comorbidity-specific risk analysis with a Pepe-Mori test of $p<0.0001$ to the disadvantage of the RTx file (EBRT $60.4 \% / 27.1 \%$ RPE). For the subgroup of high-risk tumors, a more effective local tumor control based on RPE can be found in comparison to EBRT, without being able to show a significant difference.

Conclusions: It was possible to prove that the therapy decision between two guideline-covered, alternative therapy recommendations for the localized and locally advanced PCA can be made rationally based on defined variables and categories in a private consultation. Based on our results in the RPE arm at lower PSA, among younger patients, with a rather low D'Amico score, lower Charlson score, higher Gleason score and higher prostate volume. This means our outcome comparisons are permissible and

Citation: Boehm WDU, Koch R, Froehner M, Wenzel S, Werner C, et al. (2020) Radical Prostatectomy versus External Beam Radiotherapy - Indication and the Way to the Decision: A Cumulative Non-Interventional Unicentric Retrospective Trial of about 20 Years. Int Arch Urol Complic 6:069. doi. org/10.23937/2469-5742/1510069

Accepted: April 04, 2020: Published: April 06, 2020

Copyright: (C) 2020 Boehm WDU, et al. This is an open-access article distributed under the terms of the Creative Commons Attribution License, which permits unrestricted use, distribution, and reproduction in any medium, provided the original author and source are credited. 
are not falsified by subjective allocations. The analysis of prognosis risks in the variables may underpin the value of the clinical view with a high level of evidence.

Patient summary: In this observation trial, it was possible to provide proof that the worse outcome of the EBRT file in overall survival for all risks is also decisive due to the higher comorbidity risk; For tumor-specific survival no significant difference between the two therapy arms could be proven. For the subgroup of high-risk tumors, however, we were able to demonstrate a survival advantage for the RPE file, but without reaching the level of significance in the process.

\section{Keywords}

Prostate cancer, Preference decision RPE vs. EBRT, Survival analysis

\section{Abbreviations}

RPE: Radical Prostatectomy; EBRT(RTx): External Beam Radiotherapy; DES: Diethylstilbestrol; GCP: Good Clinical Practice, OS: Overall Survival

\section{Introduction}

With localized prostate cancer [3], external beam radiotherapy conforms to the guidelines as an equivalent recommended option for prostatectomy for the initial therapy $[1,2]$. With regard to the multivariable of prostate cancer and the comorbidity of older patients, this recommendation continues to be active, although sometimes controversial $[1,4,5]$. In addition, regionally divergent indications can be further demonstrated $[6,7]$. "To date, there is no level 1 evidence comparing the efficacy of RPE and radiotherapy for patients for clinically-localized prostate cancer" [8].
The different weighting of the variables that determine the indication in daily practice, such as the risks included for a bias depending on the design and stratification of controlled studies, turns out to be problematic, in particular for the direct comparison of the RPE ad-hoc therapy with EBRT in combination with temporary androgen deprivation. Also, because it is very difficult to assess the radiation in EBRT monitoring biopsies based on a therapeutic principle primarily intended to be retarded due to histopathological distinctions of treatment effects in the regression trend, with regard to residual/recurrent areas, as well as in change of grading $[9,10]$.

\section{Patients and Methods}

\section{Description of the clientele}

During the observation period from 1996-2016, at a private consultation for urology 1,166 patients were diagnosed with prostate cancer and the primary treatment option was decided on based on grading and staging. As such, $47.7 \%$ were assigned to radical prostatectomy, $22.0 \%$ to external beam radiotherapy, $5.1 \%$ to active surveillance from 2008 and only $0.5 \%$ to alternative procedures with a curative intention. The proportion of patients who could only be assigned to a primarily palliative setting was $21.3 \%$ (Figure 1 ).

From the pool of all the recorded prostate cancers at the private consultation (Figure 1) we selected for this examination in the period 1996-2016, following their instruction and written consent for the scientific use and storage of data, those 465 patients, who were cumula-

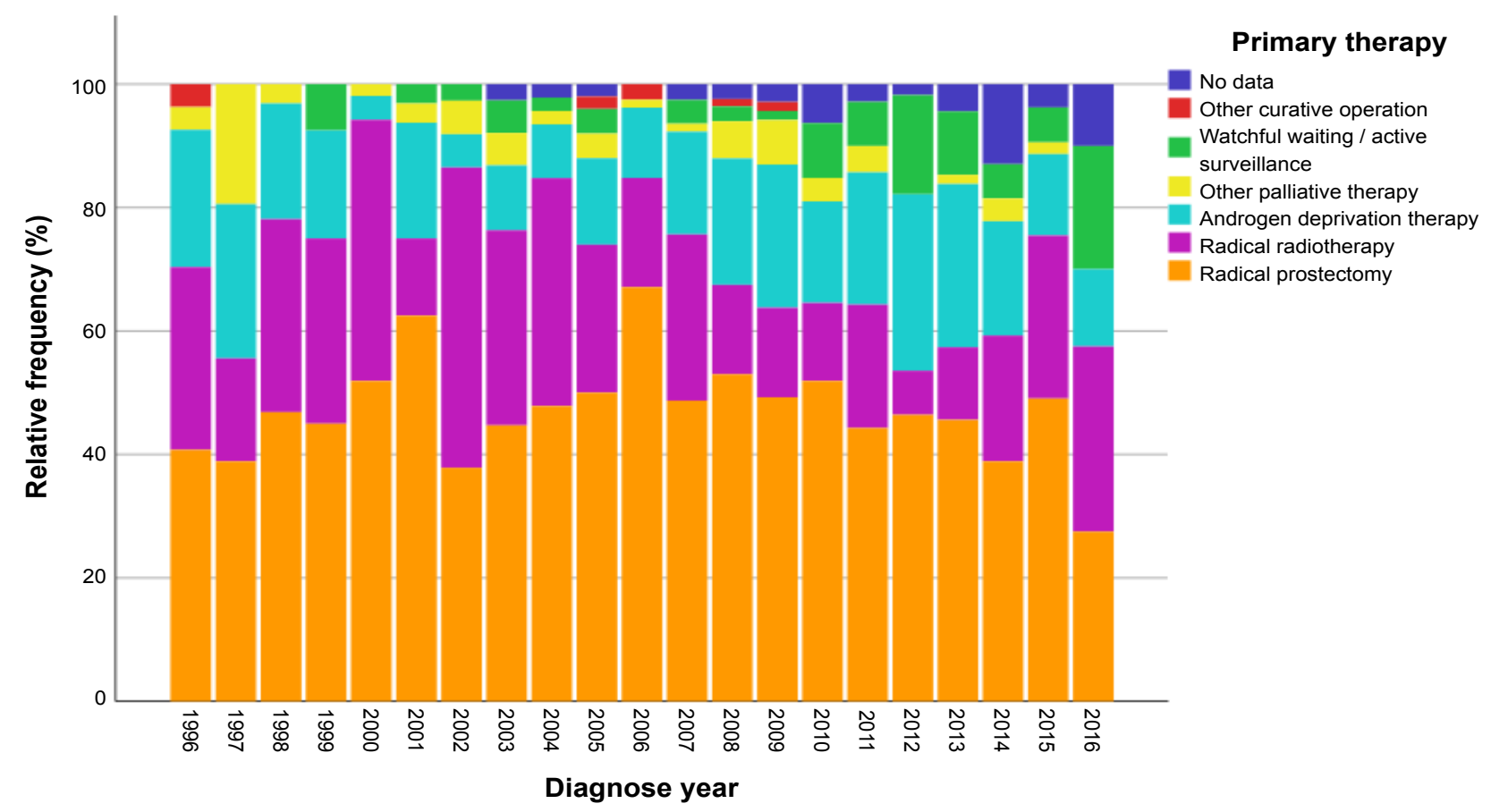

Figure 1: Spectrum of total primary treatment decisions at our private consultation over 20 years*.

*Deferred and defensive treatment strategies in the strict sense of active surveillance were not yet accepted in Germany as a curative option for localized PCA in 1996 and only gained an initial national response in 2008 through Lothar Weissbach with the HAROW study he initiated [16] plus the S3 guideline 2009, version 1.00 of the DGU [17]. 


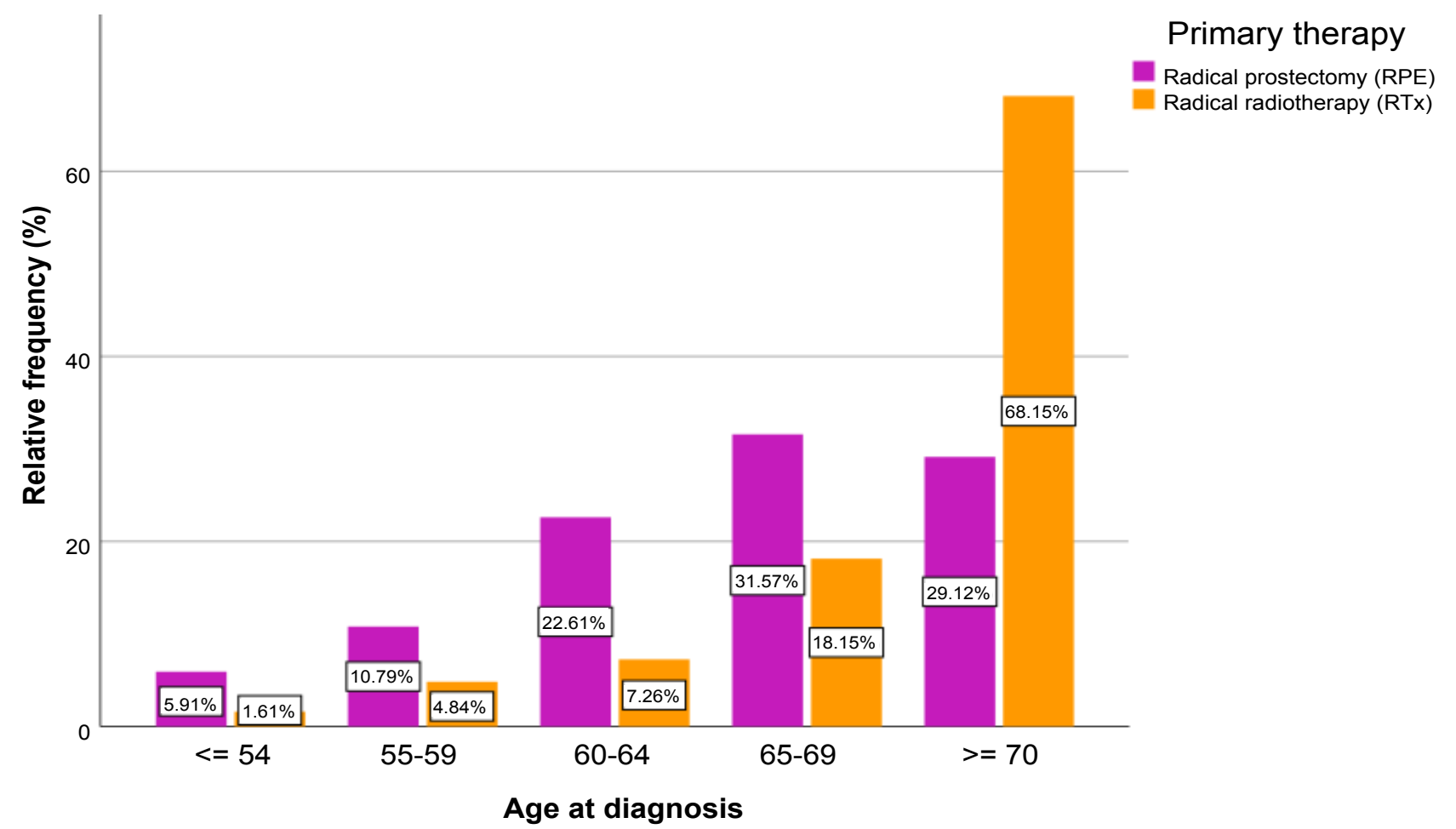

Figure 2: Age at diagnosis stratified for the option of therapy.

tively enrolled chronologically in the RPE file following the initial consultation, as well as a further 234 patients in the EBRT file who were diagnosed with a localized tumor.

We found the median ages for the RPE arm were 67.00 years (standard deviation 6.52) and for the EBRT arm 73.00 years (standard deviation 6.56). Retrospectively, even in advance of conditions in practice, a prioritized coincidence between the therapy decision and the patients' ages at diagnosis was recognizable (Figure 2).

In terms of surgery, the open RPE was retrospectively dominant as expected before the robot-assisted retropubic radical prostatovesiculectomy, which was introduced later on $90.1 \%$ of these interventions took place at the university's high-volume center in Dresden. The primarily curative intended percutaneous radiotherapy was administered with total dosages of 70-78 Gy, in individual doses of 1.8 to $2.0 \mathrm{~Gy}$ 3D-conform/IMRT and CT-planned at two centers. The total doses applied increased in line with the guidelines during the study period.

Based on the D'Amico classification, 4\% of the patients from the low-risk group (24.1\%) were treated neoadjuvant with a classic antiandrogen for three months, $47.2 \%$ of the intermediate risk group (33.7\%) and all the patients in the high-risk group (42.2\%) adjuvant with the $\mathrm{GnRH}$ analog leuprorelin for two or three years. In 29 cases, the histopathological assessment of the WHO grading system still practiced in the 1990s had to be merged with the Gleason grading system. WHO grade $1 \mathrm{a} / 1 \mathrm{~b}$ tumors to Gleason score 6 , WHO grade $2 \mathrm{a} / 2 \mathrm{~b}$ tumors to Gleason $7 \mathrm{a} / 7 \mathrm{~b}$ and $\mathrm{WHO}$ grade 3 tu- mors to Gleason 8/10. Comorbidity was recorded and classified in accordance with the Charlson comorbidity index [11].

The collected data were sent anonymized to the Regional Clinic Cancer Registry, cross-checked there and made partially available to the Common Epidemiology Cancer Registry in Berlin. Fifty-five clinically specific items in the EBRT file and 42 items in the RPE file were documented continuously in the inpatient registry. End of the following on December 31, 2016.

\section{Purpose of this study}

The data analysis should answer the question whether the individualized RPE versus EBRT therapy decision can be retrospectively justified in the case of localized or locally advanced PCA without ideal, selective limitations. It was particularly important to us primarily to investigate in addition to the univariate survival analyses the confounder-adjusted comparison of the tumor-specific and comorbidity-specific mortality rates between the RPE and EBRT. An associated retrospective consideration of the known side-effects was not a subject of this work.

\section{Statistical methods}

Univariate comparisons of both therapy arms: Covariables that were used to characterize the therapy alternatives were examined using Mann-Whitney-U tests and chi square tests. The outcome was determined by Kaplan-Meier estimates for total mortality (OS) and the cumulative mortality rates (CMR) of the two competing risks-tumor specificity and comorbidity. In addition, Mantel-Haenszel estimates of hazard ratios (HR), log- 


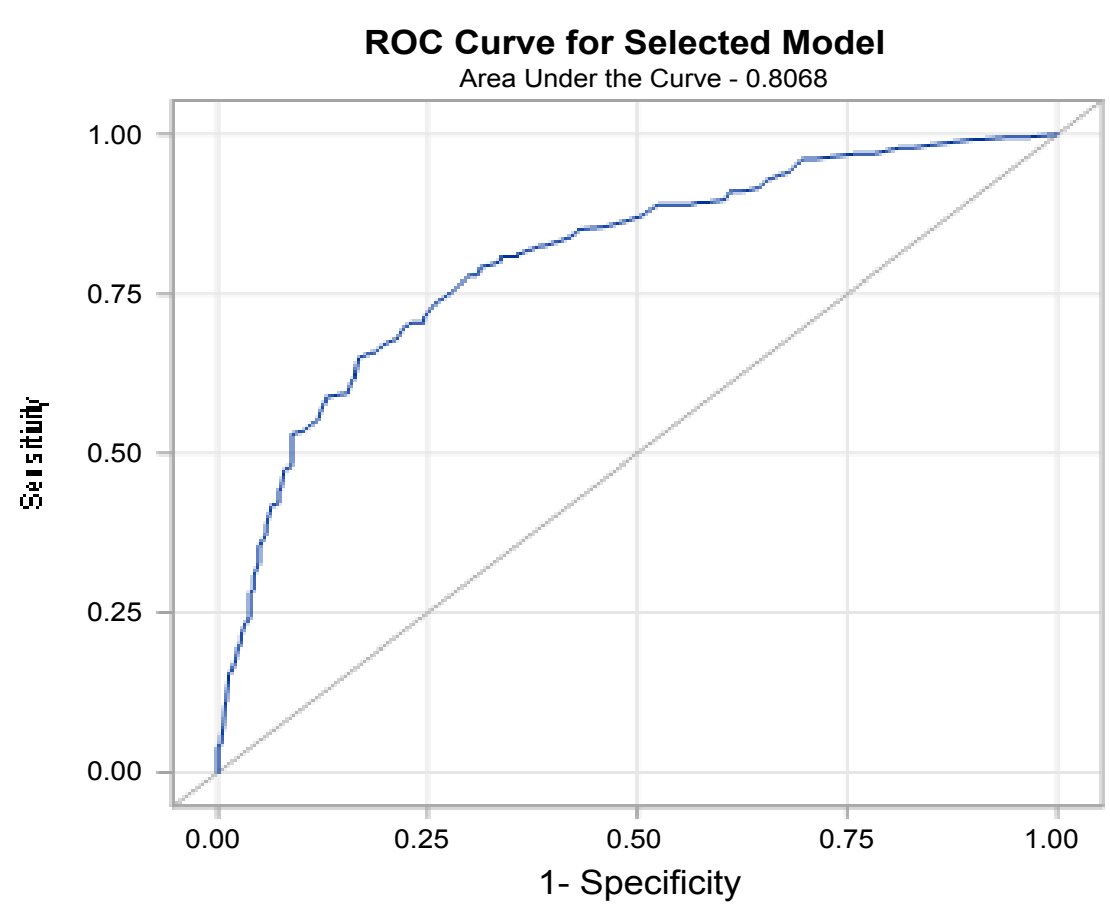

Figure 3: Receiver operating curve of the logit model.

Table 1: Characteristics of study group before therapy in a univariate comparison.

\begin{tabular}{|l|l|l|l|}
\hline Variable & Mean (SD) or percent RPE & Mean (SD) or percent ERBT & p value \\
\hline Age & $66.6(6.52)$ & $72.55(6.56)$ & $<0.0001$ \\
\hline Proportion pos. probes & $0.40(0.27)$ & $0.36(0.14)$ & 0.4612 \\
\hline Charlson comorbidity index & $0.68(0.90)$ & $1.14(1.09)$ & $<0.0001$ \\
\hline D'Amico score & $2.88(1.44)$ & $2.52(1.34)$ & 0.0004 \\
\hline Gleason score & $5.79(1.61)$ & $6.68(1.14)$ & $<0.0001$ \\
\hline PSA & $11.14(12.64)$ & $12.62(15.20)$ & 0.4339 \\
\hline Prostate volume & $42.95(20.22)$ & $30.01(17.57)$ & $<0.0001$ \\
\hline Stage cT1a-cT1c - variable 1 & $60.8 \%$ & $39.2 \%$ & $<0.0001$ \\
\hline cT2a-cT2b - variable 2 & $67.7 \%$ & $32.3 \%$ & \\
cT2c - variable 3 & $78.1 \%$ & $21.9 \%$ & \\
cT3a-cT3b - variable 4 & $35.7 \%$ & $64.3 \%$ & \\
\hline
\end{tabular}

rank tests (OS) and Pepe-Mori tests were used for comparison. The same methodology was used for estimating the cumulative incidence rates for relapse. As such, death from the tumor was also considered a relapse and death from another cause a competing event.

Objectivation of the therapy allocation: Multivariate logistical regression models with step-by-step model selection were used in order to be able to more objectively assess the separability of the two forms of therapy based on the covariables.

Confounder-adjusted comparison of survival times: Stratified estimates of hazard ratios in multivariate proportional hazard models ( $\mathrm{PH}$ models) are based on strata that result from propensity matching with regard to the allocation of patients to the two therapy alternatives RPE vs. EBRT. As such, the logit of the propensity score was used, which arises from the second resulting multi- variate logit model (Figure 3) for therapy allocation. Optimal variable rating was used as a match strategy and the logit of the propensity score as a distance measure. These multivariate analyses were carried out for both the OS as well as for CMRs of the competing risks.

All the analyses were completed with the statistical software SAS V9.4 [12].

\section{Results}

Of the 742 enrolled patients at our practice, 28 patients from the RPE therapy group and 15 patients from the EBRT therapy group EBRT were excluded, as in one or more of the variables used MAR (missing at random) was noticed - assumed by the author without further justification. The examined characteristics (variables) are presented in Table 1.

We encountered the conceivable deficit of the 


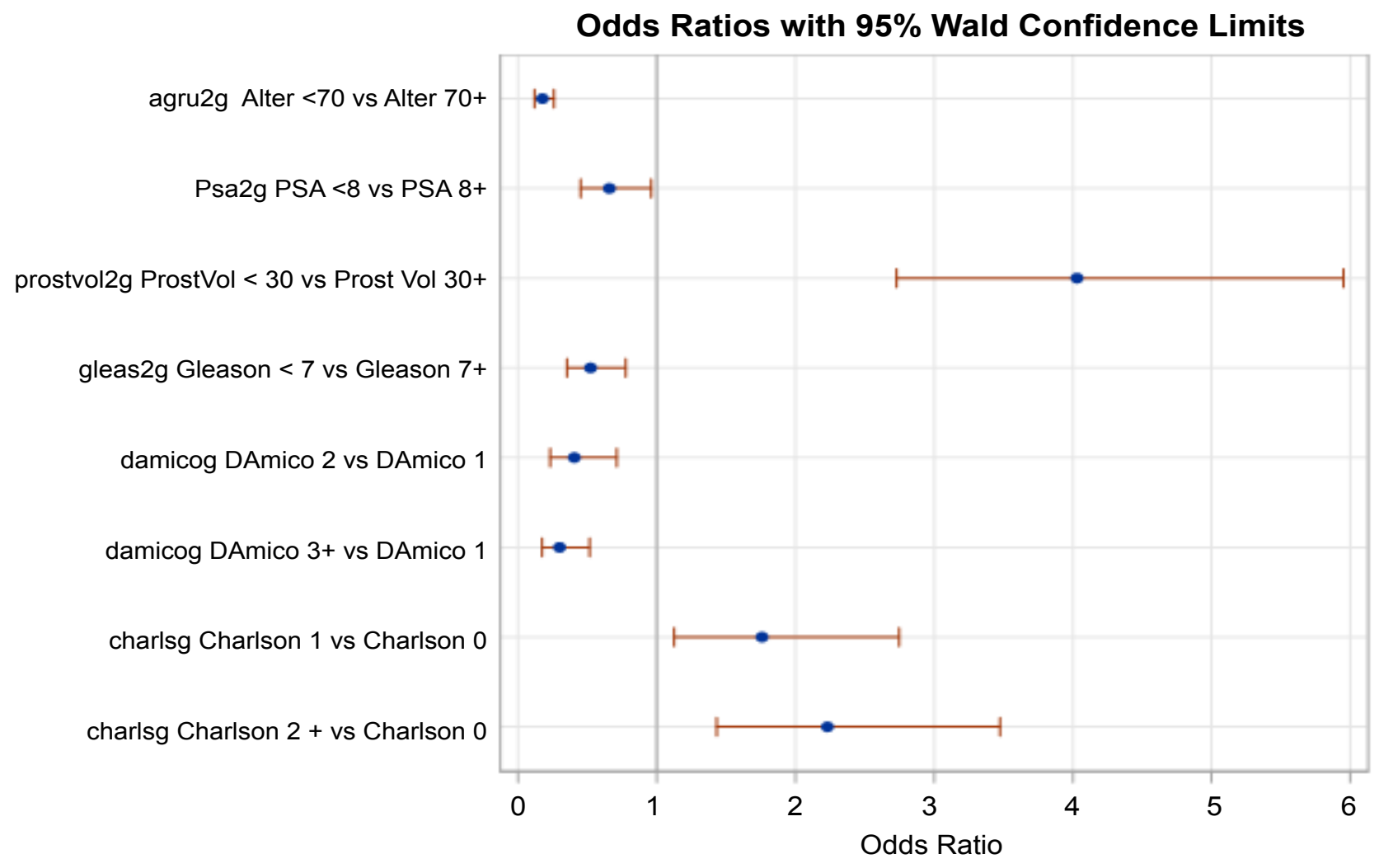

Figure 4: Forest plot of the odds ratios of the logit model (ORs and 95\% confidence intervals).

*Stage cT1a-cT1c (1), stage cT2a-cT2b (2), stage cT2c (3), stage cT3a-cT3b (4).

Table 2: Logit model for the allocation to EBRT group (OR < means, RPE preferred).

\begin{tabular}{|l|l|l|}
\hline Variable & OR & p value \\
\hline Intercept & & 0.1925 \\
\hline PSA (ref. < 8) 8+ & $1.52(1.04-2.21)$ & 0.03 \\
\hline Prost. vol. (ref. < 30) 30+ & $0.25(0.17-0.37)$ & $<0.0001$ \\
\hline Gleason score (ref. < 7) 8+ & $1.91(1.29-2.84)$ & 0.0013 \\
\hline D'Amico (ref. 0-1) 2 & $0.42(0.23-0.71)$ & 0.0017 \\
\hline 3+ & $0.30(0.17-0.52)$ & $<0.0001$ \\
\hline Charlson index (ref. 0) 1 & $1.76(1.13-2.75)$ & 0.0131 \\
\hline $2+$ & $2.23(1.43-3.48)$ & 0.0004 \\
\hline Age (ref. < 70) 70+ & $5.70(3.85-8.43)$ & $<0.0001$ \\
\hline
\end{tabular}

non-initiated randomization when checking the allocations made to the two alternative therapy arms for objectivity and reproducibility by developing a multivariate logit model (Figure 3). Satisfactory traceability of the retrospective allocation (c-statistic $=0.868, p=$ 0.0711 in the Hosmer-Lemeshow adaptation test) was therefore achieved.

In order to rule out an instinctive momentum, which would have a decisive influence on the applied allocation of patients to therapy group RPE or EBRT, a statistically valid logistical regression model was developed (Table 2), both including and without the variables considered in Table 1 as univariate.

The odds ratios of the logit model (Figure 4) allow for the following conclusions.
- The allocation in this study was carried out objectively and comprehensibly.

- The EBRT group was more likely to be allocated with higher PSA, lower prostate volume, a higher Gleason score, a usually lower D'Amico score, a higher Charlson score and at clinical stage cT3acT3b as well as patients over the age of 70 . In contrast to the univariate test, the variable PSA was now statistically relevant.

Overall survival (OS) and cumulative mortality rates (CMR) for the two competing therapy options are stated in Table 3.1 and Table 3.2. The non-parametric estimate of the allocation system's hazard ratio at $\mathrm{HR}=3.81(95 \%$ $\mathrm{Cl}: 2.74-5.31, \mathrm{p}=0.0001$ ) also shows a clear advantage for the RPE therapy option.

The further breakdown of the total and therefore the mortality rates also dependent on comorbidity shows comorbidity clearly as a cause of the discrepancy (Pepe-Mori test $p=0.0001$ ), in contrast to the analysis of tumor-specific mortality (Pepe-Mori test $p=0.2122$ ). The comparison of the models therefore allows these conclusions to be made.

In the multivariate analysis, there are no tumor-specific differences between both therapy groups either. Instead, the differences are only caused by age, the Charlson score, and due to the rate of the positive biopsy cylinders. The Charlson comorbidity score determines the diametral hazard ratio for the tumor-specific and comorbidity-specific mortality rates. Further ex- 
Table 3.1: Overall survival (OS) and cumulative mortality rates (CMR) for both competing risks concerning

\begin{tabular}{|l|l|l|l|}
\hline Years & OS & CMR tumor specific $\mathbf{( 9 5 \% C I ) ~}$ & CMR Comorbidity specific (95\%CI) \\
\hline 2 & 96.7 & $0.0(0.0-0.0)$ & $3.3(1.1-5.6)$ \\
\hline 4 & 89.5 & $0.5(0.0-1.5)$ & $10.0(6.0-14.0)$ \\
\hline 6 & 79.6 & $2.6(0.3-4.8)$ & $17.8(12.6-23.1)$ \\
\hline 8 & 69.4 & $5.4(2.1-8.6)$ & $25.2(19.1-31.4)$ \\
\hline 10 & 60.0 & $8.7(4.4-13.0)$ & $31.0(24.4-38.1)$ \\
\hline 12 & 50.9 & $11.3(6.2-16.4)$ & $37.8(30.1-45.5)$ \\
\hline 14 & 41.3 & $15.8(9.3-22.2)$ & $42.9(34.3-51.5)$ \\
\hline 16 & 31.5 & $15.8(9.3-22.2)$ & $52.7(42.6-62.9)$ \\
\hline 18 & 19.2 & $20.5(11.7-29.3)$ & $60.4(47.3-73.5)$ \\
\hline 20 & 19.2 & $20.5(11.7-29.3)$ & $60.4(47.3-73.5)$ \\
\hline
\end{tabular}

therapy. group RPE (20 years).

Table 3.2: Overall survival (OS) and cumulative mortality rates (CMR) for both competing risks concerning therapy group EBRT.

\begin{tabular}{|l|l|l|l|}
\hline Years & OS & CMR tumor specific $\mathbf{( 9 5 \% C I )}$ & CMR Comorbidity specific (95\%CI) \\
\hline 2 & 97.7 & $0.6(0.0-1.4)$ & $1.7(0.50-2.8)$ \\
\hline 4 & 94.6 & $1.6(0.4-2.7)$ & $3.8(2.0-5.6)$ \\
\hline 6 & 92.2 & $2.1(0.7-3.5)$ & $5.7(3.5-8.0)$ \\
\hline 8 & 88.2 & $3.4(1.5-5.2)$ & $8.4(5.6-11.3)$ \\
\hline 10 & 84.3 & $4.2(2.0-6.3)$ & $11.6(8.0-15.2)$ \\
\hline 12 & 81.1 & $6.0(3.1-9.0)$ & $12.8(8.9-16.8)$ \\
\hline 14 & 76.1 & $9.3(5.0-13.5)$ & $14.6(10.0-19.2)$ \\
\hline 16 & 69.0 & $11.4(6.3-16.4)$ & $19.6(13.5-25.7)$ \\
\hline 18 & 61.7 & $13.7(7.0-20.5)$ & $24.6(16.6-32.5)$ \\
\hline 20 & 56.8 & $16.2(8.1-24.4)$ & $27.1(18.0-36.1)$ \\
\hline
\end{tabular}

Table 4.1: Proportional hazard models for overall survival.

\begin{tabular}{|c|c|c|c|c|c|c|}
\hline \multirow{2}{*}{ Variable } & \multicolumn{2}{|l|}{ RPE } & \multicolumn{2}{|l|}{ EBRT } & \multicolumn{2}{|l|}{ RPE + EBRT } \\
\hline & HR (95\%Cl) & $P$ value & HR $(95 \% \mathrm{Cl})$ & p value & HR (95\%CI) & p value \\
\hline $\mathrm{PSA}<7$ & & & 1 (ref.) & & 1 (ref.) & \\
\hline $7+$ & & & $1.60(1.07-2.40)$ & 0.0216 & $1.43(1.05-1.95)$ & 0.0238 \\
\hline Gleason 0-6 & & & 1 (ref.) & & 1 (ref.) & \\
\hline $7+$ & & & $1.94(1.26-2.99)$ & $0.00<1$ & $1.56(1.12-2.17)$ & 0.0084 \\
\hline D'Amico 0-1 & 1 (ref.) & & & & 1 (ref.) & \\
\hline 2 & $1.76(0.75-4.12)$ & 0.1957 & & & $1.28(0.80-2.07)$ & 0.3057 \\
\hline $3+$ & $3.50(1.55-7.93)$ & 0.0027 & & & $1.72(1.09-2.71)$ & 0.0192 \\
\hline Age $<70$ & 1 (ref.) & רחח & & & 1 (ref.) & \\
\hline $70+$ & $2.17(1.33-3.55)$ & & & & $1.65(1.16-2.34)$ & \\
\hline Therapy group RPE & & & & & 1 (ref.) & \\
\hline RTx & & & & & $2.71(1.93-3.81)$ & $<0.0001$ \\
\hline
\end{tabular}

planatory variables for the comorbidity CMRs are the age and the therapy group; the latter are considerably less favorable for the EBRT compared to the RPE.

Furthermore, the multivariate models for the two alternative therapy arms were analyzed separately. We are well aware of the relatively lower number of cases that result as a risk in the assessment. On the other hand, it is also apparent from the separate analyses (Table 4.1) that several of the explanatory variables are only specifically important in one of the two groups such as age only in the EBRT group.

The tumor-specific mortality is predicted solely by the D'Amico risk classes in accordance with the confounder adjustment in the RPE arm, in the RTx arm solely by the age group. This consequence confirms the clinically exercised procedure in practice. Only when the two therapy arms are merged do the Charlson categories appear more substantial (Table 4.2). 
Table 4.2: Proportional hazard models for tumor-specific survival.

\begin{tabular}{|c|c|c|c|c|c|c|}
\hline \multirow{2}{*}{ Variable } & \multicolumn{2}{|l|}{ RPE } & \multicolumn{2}{|l|}{ EBRT } & \multicolumn{2}{|l|}{ RPE + EBRT } \\
\hline & HR (95\%Cl) & $p$ value & HR (95\%Cl) & p value & HR (95\%Cl) & p value \\
\hline$P S A<7$ & & & & & 1 (ref.) & \\
\hline $7+$ & & & & & $1.89(1.0-3.58)$ & 0.0519 \\
\hline D'Amico 0-1 & 1 (ref.) & & & & 1 (ref.) & \\
\hline 2 & $1.37(0.27-6.88)$ & 0.7044 & & & $1.07(0.40-2.85)$ & 0.8984 \\
\hline $3+$ & $4.87(1.16-20.52)$ & 0.0311 & & & $2.48(1.04-5.96)$ & 0.0417 \\
\hline Charlson 0 & & & & & 1 (ref.) & \\
\hline 1 & & & & & $0.46(0.22-0.95)$ & 0.0363 \\
\hline $2+$ & & & & & $0.34(0.14-0.83)$ & 0.0173 \\
\hline Age $<70$ & & & 1 (ref.) & 0 & 1 (ref.) & \\
\hline $70+$ & & & $0.35(0.16-0.79)$ & 0.018 & $1.65(1.16-2.34)$ & \\
\hline Therapy group RPE & & & & & 1 (ref.) & \\
\hline RTx & & & & & $2.41(1.30-4.48)$ & 0.0053 \\
\hline
\end{tabular}

Table 4.3: Proportional hazard models for comorbidity specific survival.

\begin{tabular}{|c|c|c|c|c|c|c|}
\hline \multirow{2}{*}{ Variable } & \multicolumn{2}{|l|}{ RPE } & \multicolumn{2}{|l|}{ EBRT } & \multicolumn{2}{|l|}{ RPE+EBRT } \\
\hline & HR (95\%Cl) & $\mathbf{p}$ & HR (95\%Cl) & $\mathbf{p}$ & HR (95\%Cl) & $\mathbf{p}$ \\
\hline$P S A<7$ & & & & & 1 (ref.) & \\
\hline $7+$ & & & & & $1.89(1.0-3.58)$ & 0.0519 \\
\hline Gleason 0-6 & & & 1 (ref.) & 0,0532 & & \\
\hline $7+$ & & & $1.65(1.0-2.75)$ & 0.0503 & & \\
\hline Charlson 0 & & & 1 (ref.) & & 1 (ref.) & \\
\hline 1 & & & $2.18(1.03-4.60)$ & 0.0416 & $1.64(1.02-3.77)$ & 0.0394 \\
\hline $2+$ & & & $3.28(1.71-6.30)$ & 0.0004 & $2.43(1.57-3.77)$ & $<0.0001$ \\
\hline Age $<70$ & 1 (ref.) & & 1 (ref.) & & 1 (ref.) & \\
\hline $70+$ & $2.39(1.33-4.30)$ & & $1.94(1.08-3.51)$ & 0.0274 & $2.14(1.41-3.25)$ & 0.0004 \\
\hline Therapy group RPE & & & & & 1 (ref.) & \\
\hline $\mathrm{RTx}$ & & & & & $2.22(1.51-3.28)$ & $<0.0001$ \\
\hline
\end{tabular}

The comorbidity-specific mortality is predicted, in accordance with the confounder adjustment in the RPE arm, solely by age on the other hand; in the EBRT arm in addition by the Gleason and Charlson score. By merging the two groups, a clearly elevated PSA value also becomes significant, whereas the Gleason classification appears less important. As such, the confounder-adjusted superiority of the RPE over the EBRT is clearly demonstrated by the HR = 2.2 (Table 4.3).

From the propensity-matched therapy comparisons, it is clear that the confounder-adjusted superiority of the RPE is clearly confirmed both in terms of overall survival as well as in tumor-specific mortality rates. In addition, the D'Amico score is significant from this perspective both in terms of overall survival as well as in specific mortality rates. This applies in the same way for the Gleason as well as the Charlson score. The influence of age on the specific mortality rates can no longer be proven (Table 4.4). This could be an indication that the obviously age-dependent allocation on the results has an impact with a simpler methodology.
Ultimately, however, age is only significant for mortality due to comorbidity but not for tumor-specific mortality and overall survival.

\section{Discussion}

The heterogeneous tumor biology of the PCA is accepted today as much as the superior dynamic of the variables that determine the prognosis - up to the prostate volume [13]. In the pre-therapeutic risk classification, the navigational use of the nomograms first published by Kattan [14] in 1997 prove to be clinically interesting; even more clearly in the later, updated version [15] but without being regularly used in practice later on. On the other hand, the discussion of the ideal therapy option for localized tumors in a curative setting in the area of debate between tumor aggressiveness and the patient's survival probability persists [16]. In particular, there is still no confirmed reproducible proof of which therapy option should be favored in the event of a high-risk tumor without excluding comorbidity $[5,8,17$ 19]. Especially as there is also a high risk of allocating older-but still healthy for their age - high-risk patients 
Table 4.4: Proportional hazard models basing on propensity matching.

\begin{tabular}{|c|c|c|c|c|c|c|}
\hline \multirow{2}{*}{ Variable } & \multicolumn{2}{|l|}{ Overall survival } & \multicolumn{2}{|c|}{ Tumor specific mortality } & \multicolumn{2}{|c|}{ Comorbidity specific mortality } \\
\hline & HR $(95 \% \mathrm{Cl})$ & p value & HR $(95 \% \mathrm{Cl})$ & p value & HR (95\%Cl) & p value \\
\hline Gleason 0-6 & & & 1 (ref.) & & & \\
\hline $7+$ & & & $2.86(1.18-6.91)$ & 0.0199 & & \\
\hline D'Amico 0-1 & 1 (ref.) & & 1 (ref.) & & 1 (ref.) & \\
\hline 2 & $3.29(1.29-8.40)$ & 0.0127 & $0.75(0.23-2.47)$ & 0.6352 & $6.03(2.74-13.29)$ & $<0.0001$ \\
\hline $3+$ & $5.81(2.32-14.54)$ & 0.0002 & $4.40(1.23-15.72)$ & 0.0222 & $6.97(3.08-15.75)$ & $<0.0001$ \\
\hline Charlson 0 & & & & & 1 (ref.) & \\
\hline 1 & & & & & $0.94(1.34-4.89)$ & 0.8673 \\
\hline $2+$ & & & & & $2.56(1.34-4.89)$ & 0.0045 \\
\hline Age $<70$ & & & & & 1 (ref.) & \\
\hline $70+$ & & & & & $2.50(1.14-5.50)$ & 0.0229 \\
\hline Therapy group RPE & 1 (ref.) & & 1 (ref.) & & 1 (ref.) & \\
\hline $\mathrm{RTx}$ & $3.05(1.89-4.92)$ & $<0.0001$ & $2.27(1.05-4.91)$ & 0.0366 & $2.15(1.31-3.54)$ & 0.0025 \\
\hline
\end{tabular}

to less than adequate treatment [6]. In our study, we found for the $>70$ age group $29.12 \%$ of patients were allocated to the RPE arm (median age 67.00) and 78.15\% to the EBRT arm (median age 73.00). A very comparable distribution of the two therapy arms across these ages is also found by Bechis SK, et al. In an evaluation of the Capsure database, they demonstrated that in a careful stratification of older patients and consideration of comorbidity and risk, this group had a $48 \%$ reduction in mortality compared with those treated conservatively [20].

Even under the conditions of a private practice with a close personal connection with patients, the weighting of relevant variables in individual consultations and the recommendation to determine one of the competing therapies remains difficult; even though in the course of the study in addition to the improvement in oncological therapy quality, the adverse events have also gradually proven to be regressive.

Observation as well as registration studies are an attempt to map inter alia the safety and clinical effectiveness of the RPE in a direct comparison with the EBRT under conditions in practice. Thereby dispensing with any adjustment and any design in the formulation of inclusion and exclusion criteria, as is regularly practiced in the course of major randomized clinical trials and meta-analyses $[5,8,17,18]$.

The individualized initial determination of therapy is crucial for the patient's tumor-specific outcome. In order to counter arbitrary courses of treatment, this recommendation is also made in Germany more often by the interdisciplinary pretherapeutic board of a regional prostate cancer center. The recommendation should be based primarily on the complex evaluation of the different variables and the inclusion of objective study data and be less eminence-based. This is why tumor board decisions are not always reproducible [5].
In a univariate comparison as well as retrospectively in our observation trial, a stringent allocation to the alternative, competing therapy option for the variables of age, comorbidity, Gleason, clinical stage and prostate volume is also convincingly proven. PSA ( $p 0.4339$ ), D'Amico score ( $p$ 0.0004), relation of pos. probes in biopsy ( $p$ 0.4612) did not achieve this level of allocation (Table 1).

The qualitative assessment for the evidence of this procedure was made possible due to the following observations on survival: For overall survival (OS) as well as for the cumulative mortality rate we found - with all levels of risk included in the variables - highly significant differences to the disadvantage of the EBRT arm (Figure $5 a$, Figure 5b, Table 3.1 and Table 3.2). In this, we also agree wholeheartedly with the systematic review and the meta-analysis of JD Wallis, et al. [8]. The probability of registering for a secondary remote metastasis after the RPE was in our study at the 20-year follow-up stage at $0.61 \%$, and after the EBRT at $4.03 \%$. These figures correlate positively with the findings from the HAROW study and the Memorial Sloan Kettering [8,21,22].

Our own further investigations comparing the highrisk with the low/intermediate risk patients according to D'Amico in the two RPE vs. EBRT files also show that the high-risk is under much better control due to radical prostatectomy in the OS (Figure 5e and Figure 5f). We agree very much with Tritschler, et al. here [23].

In the multivariate analysis, on the other hand, clinically relevant differences are no longer found in the tumor-specific survival between the RPE vs. EBRT therapy arms (Figure $5 \mathrm{C}$ ). This finding has already been presented by Bechis SK, et al. and Hamdy, et al. [20,24]. Only when considering comorbidity-specific survival (CMR) in the two therapy arms does the finding become clear that the apparently therapeutic inferiority of the EBRT in OS (all risks) in this study is also proportionally due to the competing comorbidity/age and does not seem to 


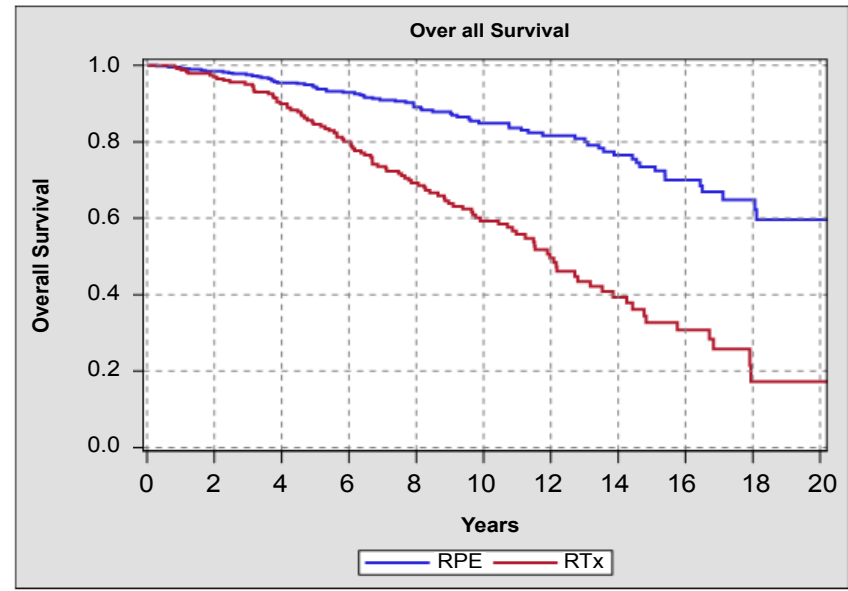

a)

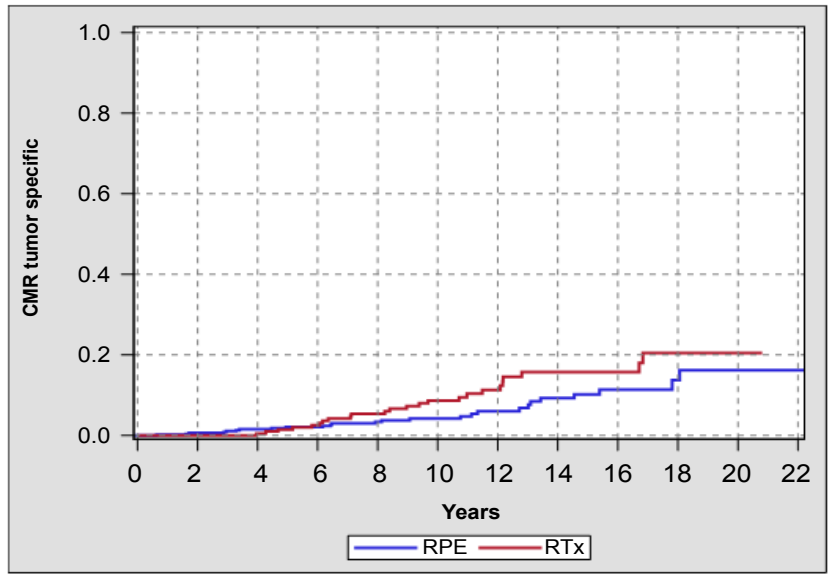

c)

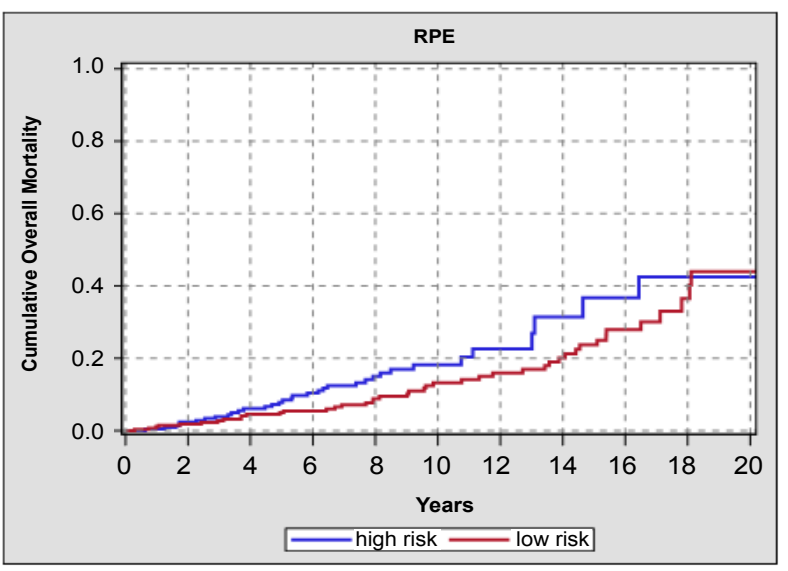

e)

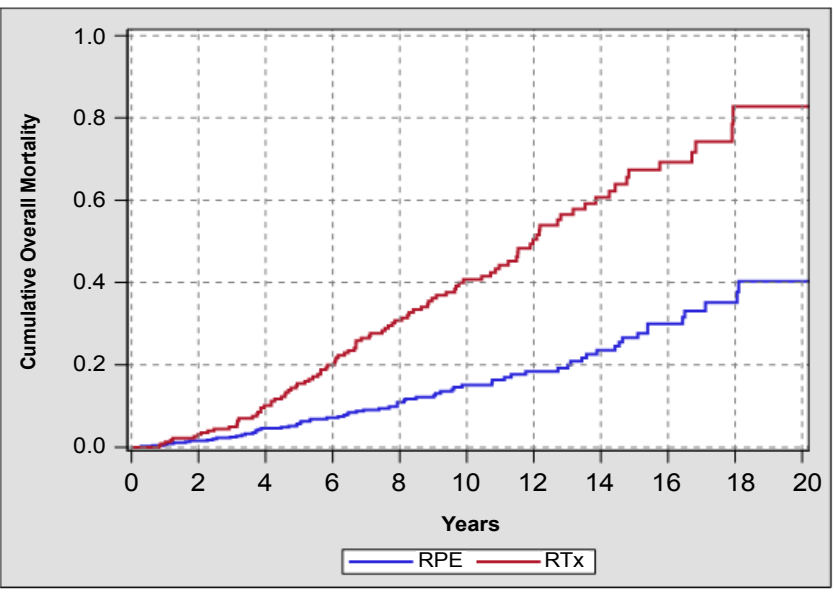

b)

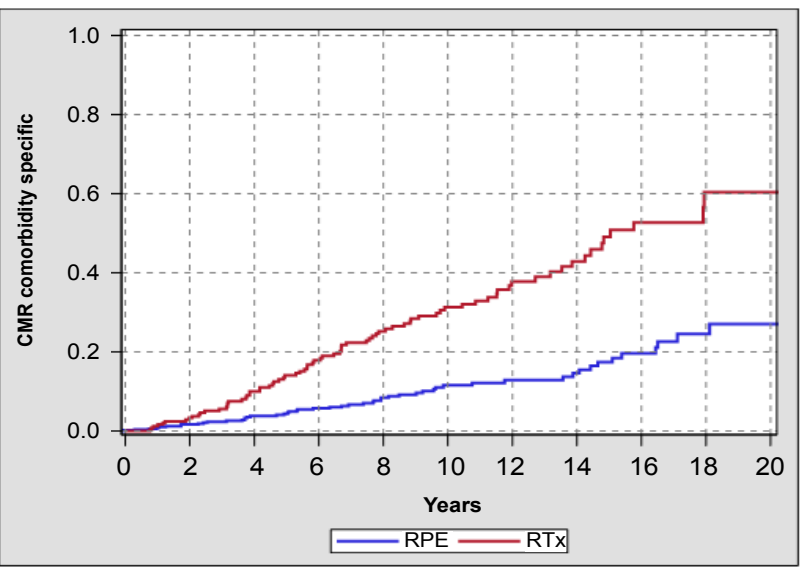

d)

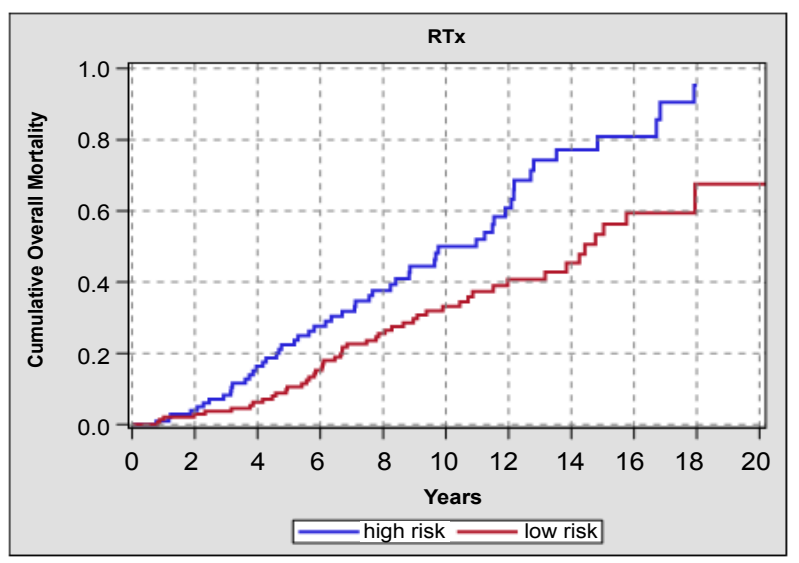

f)

Figure 5: a) Overall survival in all risks; b) Cumulative overall mortality; c) Tumor specific survival (CMR); d) Comorbidity survival rate (CMR); e) Overall mortality high risk vs. low-/intermed. risk vs. low/intermed. risk in the radical prostatectomy trial; f) Overall mortality high risk vs.low/intermed. risk in the radiotherapy trial.

be at the expense of the radiation effectiveness (Figure $5 \mathrm{~d})$. The oncologically established temporary adjuvant ADT for EBRT over 6-12 months in the intermediate and 24-36 months in the high-risk group includes conceivable cardiovascular risks at the follow-up on the other hand [25].

P Rajan, et al. also shared this view after analyzing a major observational study in Sweden for the period 1998 to 2012 [26], as well as M Fröhner and MP Wirth, who also explicitly referred to the problems in the clinical application of various comorbidity scores (ASA classification, Charlson score, CISR-G) for the therapy decision of RPE vs. RTx [27].

This observation is further supported by Berglund, et al. who investigated the complex relationship between Charlson comorbidity index, treatment and mortality in 77,536 men in the PCBaSe Sweden. In men with high-risk prostate cancer [28], radiotherapy 
was more common (range $7.7 \%$ to $21.3 \%$ ) than radical prostatectomy (range 3.0 to $11.2 \%$ ) regardless of comorbidity burden. The authors conclude that the cumulative probability of prostate cancer death given no death from competing causes was significantly higher in men with severe comorbidity in all risk groups $(p<0.01)$. This observation would ultimately mean that in this constellation a less aggressive, conservative therapy option is also chosen to help with poor general health and quality of life [17].

This clinical trend was also reflected in the reports on primary treatment of clinically localized tumors in a national cohort of the US Veterans Health Administration from 1995 to 2001 [29]. Overall survival in this cohort is also noticeably low in comparison with our findings. We can confirm mortality of over $30 \%$ after 10 years of follow-up in our analyses for the EBRT file in cumulasftive overall mortality (Figure 5b). The analyzed cohort (cT1/cT2 tumors, PSA $<20$, age $<75$ ) is characterized by a broad-based therapeutic procedure in the initial decision (surgery, radiotherapy, ADT, conservative); the authors causally excluded poor quality care as unlikely, however.

In summary, they as we do also tend to see more substantial comorbidity as the reason for the poor result in overall survival.

Barbosa, et al. conclude that the better Pivot results cannot be applied generally to the clinical reality in practice [29].

In a meta-analysis in 2016, a group led by CJD Wallis critically reviewed 19 international studies with high statistical power. Finally, the authors found an elevated risk of overall and prostate cancer-specific mortality in the EBRT group compared to surgery with lower to moderate risk - similar to our findings. At the same time, however, they also referred to underpowered randomized controlled trials, potential areas of bias and methodological limitations of observational studies [8].

In 2006, Atakura, et al. presented an initial randomized multicenter study for the clinical stage cT2b/cT3; 105 patients with a median follow-up of 102 months. In the process, all the patients were subject to neoadjuvant intended eight-week DES therapy before randomization. In the EBRT trial, adjuvant androgen deprivation continued with LHRH agonists vs. bilateral orchiectomy, or optionally with antiandrogens or reduced dose DES until progress. The authors found no significant differences between the two files for biochemical and clinical progression-free survival. In general, improved tumor-specific survival could be shown for the RPE trial but without achieving significance at $p=0.06$. However, an indirect cross-check with our similarly impressive data is excluded due to the diverse formulation of provisos in the study design and in the therapy program [30].

\section{Summary}

Careful pretherapeutic grading and staging, with the always critical assessment of variable comorbidity and age, enable a largely reliable allocation to be made to the individually ideal therapy option. The results of our study prove furthermore assured local tumor control both through RPE as well as EBRT in the normal mix of prostate cancer in the real clinical situation of a private urology consultation.

However, the study also shows that the primary referral of patients to the alternative therapy arms may obviously be done regularly in accordance with evidence-based preferences. To the RPE arm therefore the younger patients with lower PSA, lower Charlson index and usually but not significantly higher Gleason score with significantly increased prostate volume. However, we definitely do not see any methodological limitation here in the context of the objectives of this study.

In this observation trial, the age is in the median range (RPE 67.00 - ERBT 73.00 before the therapy decision is significantly above the statistics of randomized prospective trials $[13,18,20,22,30]$. After stratification, the inclusion of comorbidity also proves to be a decisive variable that determines overall survival.

The considered and evidence-based decision in the therapy choice of RPE vs. EBRT opens up for the patient an individualized perspective for a stage-appropriate curative procedure with an oncologically highly satisfactory outcome. In the clinically monitored follow-up, even after the allowance of salvage interventions, there were no significant differences in tumor-specific mortality between the two files after 20 years of observation.

\section{Acknowledgments}

\section{Author contributions}

Wolf-Diether U. Boehm and Rainer Koch had full access to all the data in this study and take responsibility for the integrity of the data and the accuracy of the data analysis. Study concept and design: WDUB, RK, acquisition of data: WDUB, AM, CW, analysis and interpretation of data: RK, WDUB, MF, MPW. Drafting of the manuscript: WDUB, RK; Critical revision of the manuscript for important intellectual content: MPW, MF, RK, SW; Statistical analysis: RK, AM; Administrative, technical or material support: SW. Supervision MPW, MF.

\section{Disclosure Statement}

The authors have no conflicts of interest to declare.

\section{References}

1. Heidenreich A, Bastian PJ, Bellmunt J, Bolla M, Joniau $S$, et al. (2014) EAU guidelines on prostate cancer. part 1: Screening, diagnosis and local treatment with curative intent-update 2013. Eur Urol 65: 124-137.

2. (2018) Interdisziplinäre leitlinie der qualität $S_{3}$ zur früherkennung, diagnose und therapie der verschiedenen stadien des prostatakarzinoms. AWMF. 
3. Bischoff R, Chaloupka M, Westhofen T, Grimm T, Schlenker B, et al. (2019) Current controversies in the treatment of localized prostate cancer. Urologe A 58: 524-528.

4. Barocas DA, Alvarez JoA, Resnick MJ, Koyama T, Hoffman KE, et al. (2017) Association between radiation therapy, surgery, or observation for localized prostate cancer and patient-reported outcomes after 3 years. JAMA 317: 1126-1140.

5. Heidenreich A, Böhmer D (2016) Multimodale therapie des lokal fortgeschrittenen prostatakarzinoms. Der Urologe 55: 333-344.

6. Cooperberg MR, Caroll PR (2015) Trends in management for patients with localized prostate cancer, 1990-2013. JAMA 314: 80-82.

7. Hager B, Kraywinkel K, Keck B, Katalinic A, Meyer M, et al. (2015) Integrated prostate cancer centers might cause an overutilization of radiotherapy for low-risk prostate cancer: A comparison of treatment trends in the United States and Germany from 2004 to 2011. Radiother Oncol 115: 90-95.

8. Wallis CJD, Saskin R, Choo R, Herschorn S, Kodama RT (2016) Surgery versus radiotherapy for clinically- localized prostate cancer. A systematic review and meta-analysis. Eur Urol 70: 21-30.

9. Petraki CD, Stikas CP (2007) Histopathological changes induced by therapies in the benign prostate and prostate adenocarcinoma. Histol Histopathol 22: 107-118.

10. Pilepich MV, Hederman MA (1986) Prognostic significance of the pattern of tumor regression following definitive radiotherapy for carcinoma of the prostate. Am J Clin Oncol 9: 494-499.

11. Charlson ME, Pompei P, Ales KL, MacKenzie CR (1987) A new Method of Classifying prognostic comorbidity in longitudinal studies: Development and validation. J Chron Dis 40: 373-383.

12. SAS V9.4: SAS Institute Inc., Cary, NC, USA.

13. Moschini M, Gandaglia G, Suardi N, Fossati N, Cucciara V, et al. (2015) Importance of prostate volume in the stratification of patients with intermediate-risk prostate cancer. Int $\mathrm{J}$ Urol 22: 555-561.

14. Ross PL, Scardino PT, Kattan MW (2001) A catalog of prostate cancer nomograms. J Urol 165: 1562-1568.

15. Rajan P, Sooriakumaran P, Nyberg T, Akre O, Carlsson $S$ (2017) Effect of comorbidity on prostate cancer-specific mortality: A prospective observational study. J Clin Oncol 35: 3566-3574.

16. Mottet N, Boyle H, Droz JP (2013) Decision making in elderly localized prostate cancer. In: Droz JP, Audisio R. Management of Urological Cancers in Older People. Springer, London, 163-176.
17. Berglund A, Garmo H, Tishelman C, Holmberg L, Stattin P (2011) Comorbidity, treatment and mortality. A population based cohort study of prostate cancer in PCBaSe Sweden. J Urol 185: 833-839.

18. Bill-Axelson A, Holmberg L, Garmo H, Taari K, Busch C, et al. (2018) Radical prostatectomy or watchful waiting in prostate cancer - 29-Year follow up. N Engl J Med 379: 2319-2329.

19. Lei JH, Chen YJ, Liu LR, Wei Q (2015) Radical prostatectomy and radiation therapy for high-risk prostate cancer: An update. Zhangva Nan Ke Xue 21: 663-666.

20. Bechis SK, Caroll PR, Cooperberg MR (2011) Impact of age at diagnosis on prostate cancer treatment an survival. J Clin Oncol 29: 235-241.

21. Herden J, Ansmann L, Ernstmann N, Schnell D, Weißbach $L$ (2016) The treatment of localized prostate cancer in everyday practice in Germany. A multicenter prospective observational study (HAROW) in 2957 patients. Dtsch Arztebl Int 113: 329-336.

22. Zelefsky MJ, Eastham JA, Cronin AM, Fuks Z, Zhang Z (2010) Metastasis after radical prostatectomy or external beam radiotherapy for patients with clinically localized prostate cancer: A comparison of clinical cohorts adjusted for case mix. J Clin Oncol 28: 1508-1513.

23. Tritschler S, Ganswindt U, Stief CG (2016) Localized intermediate - to high-risk prostate cancer. Urologe A 55: 318325.

24. Hamdy FC, Donovan JL, Lane JA, Malcolm M, Metcalfe C, et al. (2016) 10-Year outcomes after monitoring, surgery, or radiotherapy for localized prostate cancer. N Engl J Med 375: 1415-1424.

25. Droz JP, Albrand G, Gillessen S, Hughes S, Mottet N, et al. (2017) Management of prostate cancer in elderly patients: Recommendations of a task force of the International Society of Geriatric Oncology. Eur Urol 72: 521-531.

26. Pintilie M (2006) Competing risks: A practical perspective. Wiley, Chichester.

27. Froehner M, Wirth MP (2012) Age and comorbidity in early prostate cancer. Urologe A 51: 1356-1361.

28. Parker C (2004) Active surveillance of early Prostate cancer: Rationale, initial results and future developments. Prostate Cancer and Prostatic Dis 7: 184-187.

29. Barbosa PV, Thomas IC, Srinivas S, Buyyounouski MK, Chung BI, et al. (2016) Overall survival in patients with localized prostate cancer in the US Veterans Health Administration: Is PIVOT generalizable? Eur Urol 70: 227-230.

30. Akakura K, Suzuki H, Ichikawa T, Fujimoto $H$, Maeda O, et al. (2006) A randomized trial comparing radical prostatectomy plus endocrine therapy versus external beam radiotherapy plus endocrine therapy for locally advanced prostate cancer. Jpn J Clin Oncol 36: 789-793. 